\title{
BARRERAS Y FACILITADORES PARA LA INCLUSIÓN EDUCATIVA DE PERSONAS CON DISCAPACIDAD INTELECTUAL
}

\section{Barriers and Facilitators for the Educational Inclusion of Persons with Intellectual Disabilities}

\author{
Cristina Nieto Carmona \\ Universidad de Sevilla \\ Anabel Moriña DíEz \\ Universidad de Sevilla \\ cristinanietodoctorado@gmail.com
}

Recepción: 5 de octubre de 2020

Aceptación: 15 de febrero de 2021

Resumen: El propósito de este estudio es analizar las barreras y ayudas que 32 personas con discapacidad intelectual (DI), escolarizadas en centros ordinarios, han identificado en su trayectoria escolar. Las autoras realizaron un estudio cualitativo basado en los supuestos de la investigación inclusiva con adultos españoles con DI. Se recogieron los datos mediante entrevistas individuales y fueron analizados a través de un sistema de categorías y códigos. En el análisis colaboraron activamente los participantes en la investigación. Los resultados muestran que las barreras y las ayudas que se encontraron las personas con DI estaban relacionadas con el perfil docente, el currículo, las relaciones sociales con los compañeros y otros agentes, como la familia y los equipos directivos de los centros educativos. Como principal conclusión sobresale que la práctica docente y la relación con los iguales son fundamentales y pueden llegar a actuar tanto como barrera cuanto como ayuda. Como recomendaciones se plantean la necesidad de que los docentes se comprometan con la enseñanza de contenidos funcionales, el uso de metodologías activas y centradas en el alumnado, así como con actuaciones para promover las relaciones sociales entre el alumnado.

Palabras Clave: discapacidad intelectual; educación inclusiva; barreras; ayudas; investigación inclusiva. 
AвSTRACT: The purpose of this study is to analyse the barriers and aids that 32 people with intellectual disabilities (ID), enrolled in ordinary schools, have identified in their school experience. The authors conducted a qualitative study based on the assumptions of inclusive research with Spanish adults with ID. Data was collected through individual interviews and coded with a category system. Research participants actively collaborated in the analysis. The results show that the barriers and supports encountered by people with ID were related to the teaching profile, the curriculum, social relations with peers and other agents, such as family and school management teams. The main conclusions are that teaching practice and relationships with peers are fundamental and can act as both a barrier and a help. Recommendations include the need for teachers to be committed to teaching functional content, the use of active and student-centred methodologies, as well as actions to promote social relations between students.

KEYWORDS: intellectual disability; inclusive education; barriers; aids; inclusive research.

\section{Introducción}

L

a Declaración de Salamanca (1994) supuso un impacto no solo en el pensamiento, la política y la práctica educativa, sino también en la cultura (Ainscow et al., 2019). En el caso de las personas con discapacidad, la Agencia Europea para las Necesidades Especiales y la Educación Inclusiva (2018) y el Alto Comisionado de las Naciones Unidas reconocieron la educación inclusiva como una oportunidad para su empoderamiento (ONU, 2019), además de ser una oportunidad para eliminar las barreras al aprendizaje y la participación de todo el alumnado (Leiva, 2020; Ramberg y Watkins, 2020; Sanahuja et al., 2020). Sin embargo, en la actualidad, las prácticas de exclusión educativa y la discriminación siguen aún presentes, tanto en España como en el resto del mundo (Echeita, 2017; Lacono et al., 2019; Unesco, 2020).

Las barreras han sido definidas como obstáculos a la inclusión que dificultan o limitan el aprendizaje, pertenencia y participación activa, en condiciones de igualdad, en los procesos educativos (Ainscow, 2004). Por ejemplo, tal y como explica Messiou (2006), una persona puede experimentar exclusión cuando no puede acceder al currículo, no se le dan las oportunidades para participar en la clase, sus capacidades no son valoradas, es rechazada por sus iguales o incluso cuando se le niega el derecho de amistad. Se ha reconocido que aquellos estudiantes que experimentan estas barreras pueden ser considerados "voces escondidas” (López Melero, 2011). Por el contrario, las ayudas, apoyos of facilitadores son elementos del contexto educativo que contribuyen a que el alumnado esté incluido social y educativamente en los contextos educativos.

El propósito de este artículo es analizar las barreras y ayudas a la inclusión que personas con DI identifican cuando estuvieron escolarizados en centros ordinarios. Para ello, en la fundamentación de este trabajo se abordan cuatro temas con relación a las barreras y facilitadores a la inclusión: el docente, el currículo, los compañeros y otros agentes. 


\subsection{El docente como facilitador o barrera para la inclusión}

Los docentes pueden actuar como facilitador o barrera para los estudiantes con DI en las escuelas. Los estudios de Alesech y Nayar (2019) y Sharma et al. (2017) concluyeron que la actitud de los docentes es esencial para facilitar el aprendizaje del alumnado. Esto se traduce en el compromiso con el aprendizaje del alumnado con DI, la disposición para utilizar los recursos disponibles y la autorreflexión sobre su propia práctica. Sin embargo, Gilor y Katz (2018) plantearon que tener una actitud positiva a la inclusión no es suficiente. Los docentes necesitan impregnarse de los valores subyacentes de la inclusión para tener claros los objetivos y así poder desarrollar su práctica de forma eficaz (Low et al., 2018). Por ejemplo, los docentes no deberían enseñar con una única estrategia de enseñanza de forma genérica y homogénea para todos los estudiantes con DI ya que cada alumno presenta necesidades concretas y/o estilos de aprendizaje diferentes. Como se ha estudiado, las estrategias metodológicas deben ser diversas y ajustarse a las características del grupo (Nieto y Moriña, 2019).

Otros investigadores destacan del perfil docente las competencias personales, como autorreflexión, supervisión constante, compromiso profesional, paciencia, empatía y comprensión. Estas se identifican como valores esenciales para trabajar en aulas inclusivas (Boynton y Mahon, 2018; Gest et al., 2014).

\subsection{El currículo como facilitador o barrera para el alumnado con discapacidad inte- lectual}

Estudios recientes internacionales demuestran que las aulas inclusivas deben ser la única opción para el alumnado con DI ya que los entornos inclusivos promueven su desarrollo cognitivo y social (Nota et al., 2018; Scharenberg et al., 2019; Smogorzewska et al., 2019). Sin embargo, los resultados hallados en la revisión sistemática de Kuntz y Carter (2019) resaltan que las acciones educativas son insuficientes en las aulas inclusivas para atender a todas las necesidades. Los estudios concluyen que son necesarias para la inclusión metodologías constructivistas y centradas en el estudiante (Kurt, 2016) o el Diseño Universal para el Aprendizaje (Díez y Sánchez, 2015).

En el aula inclusiva el currículo es el eje vertebrador para garantizar el aprendizaje y la participación de todo el alumnado. Como ha definido Bolívar (2019), a través del currículo se debe garantizar el acceso a la base común de competencias clave. Es imprescindible que todos los estudiantes tengan acceso a una base común y encuentren, a la vez, la necesaria diferenciación que se ajuste a sus necesidades, estilos de aprendizaje y a la propia diversidad inherente a cualquier persona (Buchner et al., 2020).

Con relación al currículo, en el estudio de Valentim y Valentim (2019), 16 adultos con DI describieron sus trayectorias educativas. Uno de los hallazgos de este estudio fue que los estudiantes no contaron con una adaptación del plan de estudios y esto repercutió en sus resultados académicos y en el bienestar social y psicológico (frustración o autoestima). Por ello, Giangreco (2017) propone formular metas y objetivos individualizados para las personas con DI en relación con los resultados de aprendi- 
zaje de los compañeros sin DI para evitar establecer diferencias entre el alumnado. Los participantes con DI del estudio de Shogren et al. (2015) declararon que querían aprender los mismos contenidos que los demás, aunque con los recursos de apoyo necesarios (ajustes curriculares, profesionales de apoyo u otros recursos materiales). Otros estudios vieron necesaria la inclusión en el currículo de nuevos contenidos relacionados con la formación en habilidades para la vida independiente (Verdugo y Rodríguez, 2012), habilidades sociales (Díaz-Garolera et al., 2019) o educación emocional (Smith y Low, 2013).

Otro elemento del currículo que se ha descrito en los estudios como una barrera para los estudiantes con DI es la evaluación (Datta, 2013; Soulis y Floridis, 2010). Estos estudios indicaron que, con relación a la evaluación, el nivel de estrés que experimentan los estudiantes con DI fue superior al del resto de alumnado.

Por último, otros estudios se han centrado en la disposición y la ubicación del alumnado con DI dentro del aula. Por ejemplo, Van Den Berg y Stoltz (2018) mostraron que la disposición de los asientos en el aula puede ser una herramienta útil para la inclusión del alumnado. Esto es lo que Farmer et al. (2018) describen como "mano invisible del maestro".

\subsection{Los iguales, agentes clave para las personas con discapacidad intelectual}

La relación entre compañeros es un factor que determina el desarrollo académico y social de las personas con DI en las escuelas (Wang y Eccles, 2012). Los estudios destacan que las experiencias sociales positivas promueven el bienestar y la calidad de vida de los estudiantes con DI (Scharenberg et al., 2019; Smogorzewska et al., 2019). Wilson et al. (2017) constatan que las personas con DI están felices cuando se sienten incluidas y tienen amistades. En el estudio de Rushbrooke et al. (2014), los estudiantes con DI expresaron su deseo de sentirse queridos y respetados por sus compañeros de clase. Alesech y Nayar (2019) concluyeron que, para que los estudiantes logren un sentido de aceptación y pertenencia en la escuela, deben estar motivados y comprometidos con el aprendizaje. De hecho, los estudiantes sienten un mayor sentido de pertenencia cuando sus maestros los involucran en actividades cooperativas, divertidas, activas e interesantes para ellos. Es aquí donde el docente toma un papel relevante al crear ambientes que propicien las relaciones afectivas entre los estudiantes. En el estudio de Robinson et al. (2020), estudiantes con DI resaltan que evitaban ir a lugares donde no se sentían bienvenidos. Por lo tanto, ser reconocido y valorado en la escuela es fundamental para tener un sentimiento de pertenencia e identidad personal.

En este contexto, los iguales también pueden ser reconocidos como una barrera en la escuela. Las tasas de discriminación e intimidación de las personas con DI son altas en comparación con el resto de los estudiantes con otras discapacidades (Rose et al., 2016; Young et al., 2016). Estos estudiantes están más estigmatizados y sus compañeros los ven como amigos menos deseables (Valentim y Valentim, 2019). El estudio de Mañas-Olmo et al. (2020) ha evidenciado que las experiencias de discriminación y acoso en las escuelas repercutían en su conducta social y emocional, dentro y fuera de 
la escuela. Esto lleva al alumnado a una autopercepción negativa de su discapacidad e incluso a la normalización de la discriminación. Estos hallazgos sitúan a los iguales como un elemento clave en la inclusión y el desarrollo integral de los estudiantes con DI en las escuelas.

\subsection{Otros agentes clave en la inclusión de personas con discapacidad intelectual}

Otro de los agentes que las personas con DI consideran un facilitador es el personal de apoyo (Giesbers et al., 2019). Este personal puede ser cualquier profesional involucrado en el apoyo del estudiante, como los diferentes profesionales dedicados al apoyo a la diversidad de los centros educativos, monitores, personal de apoyo de otras entidades, etc. En la escuela es posible encontrar asistentes o maestros de apoyo que acompañan a los estudiantes con DI en su trayectoria académica. Desde los principios de la inclusión, estos agentes están disponibles para todos los alumnos. Sin embargo, en la mayoría de los contextos educativos están relacionados con el acompañamiento de personas con DI o con otras necesidades (Nes et al., 2017).

Investigadores como Riitaoja et al. (2019) evidenciaron que los estudiantes tienen una percepción negativa de las clases de apoyo debido a la falta de contenidos académicos que se impartían en ellas y a la división entre las clases regulares y las clases de apoyo. Sandoval et al. (2019) relacionan esta práctica con el modelo médico-terapéutico, concibiendo el aula de apoyo como un recurso diseñado exclusivamente para alumnado con DI. Sin embargo, otros estudios reconocen que el alumnado, en el aula de apoyo, encuentra un aliado: el maestro de apoyo (Boynton y Mahon, 2018; Nieto y Moriña, 2019). Esta figura se convierte en un facilitador para el alumnado con DI, ya que estos docentes demuestran habilidades de empatía, comprensión y motivación.

Por otro lado, Sharma et al. (2017) y Simón y Barrios (2019) recomiendan la necesidad de promover actitudes positivas y de colaboración entre docentes, familias y otros servicios de la comunidad para favorecer la inclusión del alumnado. El estudio de Jigyel et al. (2018) destacó la preocupación de la familia por el acoso escolar de sus hijos con DI y cuestionó la calidad del aprendizaje académico de estos en las aulas ordinarias. Con relación a esto, Acuña (2017) y Simón et al. (2016) aludieron a la necesidad de compromiso de la familia con la escuela y la confianza que estos deben depositar en la escuela.

En definitiva, teniendo en cuenta estos antecedentes, el objetivo de este artículo es analizar las barreras y ayudas con relación a la inclusión que las personas con DI han experimentado durante su escolarización en centros educativos ordinarios. A partir de ello, se pretende cubrir un hueco detectado en la literatura al escuchar a estudiantes con DI, que ha sido considerado uno de los colectivos silenciados en la escuela, y, con ello, avanzar en el conocimiento sobre la educación inclusiva. En concreto, tratamos de responder a cuatro preguntas de investigación:

1. ¿Cómo el profesorado contribuye a la inclusión del alumnado con DI?

2. ¿Qué elementos del currículo facilitan o dificultan el aprendizaje y la participación del alumnado con DI? 
3. ¿Cómo los compañeros dificultan o facilitan la inclusión del alumnado con DI?

4. ¿Qué otros agentes contribuyen a la inclusión del alumnado con DI?

\section{Método}

La metodología en la que está basada el estudio que se presenta en este artículo es cualitativa, basada en los supuestos de la investigación inclusiva (Walmsley y Johnson, 2003). En este estudio las personas con DI juegan un papel activo como coinvestigadores. En esta metodología los testimonios, en nuestro caso, de las personas con DI, para mejorar la creación de entornos educativos inclusivos son fundamentales. Además, esta metodología fomenta el empoderamiento. La investigación inclusiva supone un compromiso por evitar una jerarquía de poder entre investigadores y coinvestigadores, estableciendo una relación horizontal y una responsabilidad compartida (Nind, 2014; Vega et al., 2020). En nuestro estudio, nos comprometimos en todo momento para asegurarnos de que el estudio se llevara a cabo en una forma colaborativa. Los participantes actuaron como coinvestigadores en la elección del tema de estudio, la construcción y el análisis de las entrevistas.

Con respecto a la ética del estudio se tomaron en consideración los criterios establecidos por el Código de Ética de la Asociación Americana de Psicología (APA, 2017), en relación con la confidencialidad, el respeto a los participantes y el consentimiento informado. En concreto, para garantizar el anonimato utilizamos pseudónimos. Los participantes y los representantes legales firmaron consentimientos informados. Antes de cada entrevista explicamos a cada participante los aspectos fundamentales de este estudio para asegurarnos de que comprendían los derechos de una investigación

\subsection{Reclutamiento y participantes}

En el proceso de reclutamiento nos pusimos en contacto, a través de e-mail, con ocho centros ocupacionales de personas con DI. Dos centros respondieron y mantuvimos una reunión con los directores de cada centro ocupacional para presentar el proyecto de investigación. El reclutamiento fue el resultado de un muestreo intencional en el que los participantes debían ser hombres o mujeres adultas con DI que voluntariamente estaban dispuestos a participar en la investigación. Tuvimos una reunión en cada centro con las personas que cumplían estos criterios para solicitar su participación. Todas las personas con DI que asistieron a esta reunión se ofrecieron voluntarias para formar parte del estudio. Sin embargo, cuatro de ellos no fueron incluidos en este artículo por haber estudiado en Centros de Educación Especial (CEE). Finalmente, 32 personas participaron en el estudio (Tabla 1). 


\begin{tabular}{|c|c|c|c|c|}
\hline \multicolumn{5}{|c|}{ TABLA 1. Perfil de los participantes } \\
\hline Pseudónimo & Edad & Género & Estudios realizados & $\begin{array}{l}\text { Aula de } \\
\text { apoyo }\end{array}$ \\
\hline Ana & 25 & Femenino & Enseñanza obligatoria (ESO) & Sí \\
\hline Antonio & 31 & Masculino & $\begin{array}{l}\text { Enseñanza obligatoria (ESO) y curso de } \\
\text { marroquinería }\end{array}$ & Sí \\
\hline Álvaro & 18 & Masculino & Enseñanza obligatoria (ESO) & Sí \\
\hline Carlos & 38 & Masculino & Enseñanza obligatoria (EGB) & \\
\hline Carmen & 27 & Femenino & Enseñanza obligatoria (ESO) & \\
\hline Cristina & 27 & Femenino & $\begin{array}{l}\text { Enseñanza obligatoria (ESO) y curso de } \\
\text { jardinería }\end{array}$ & Sí \\
\hline Javi & 33 & Masculino & Enseñanza obligatoria (ESO) & Sí \\
\hline Lola & 32 & Femenino & Enseñanza obligatoria (ESO) & \\
\hline Luis & 32 & Masculino & $\begin{array}{l}\text { Enseñanza obligatoria (ESO) y curso de } \\
\text { contabilidad }\end{array}$ & Sí \\
\hline Manuela & 50 & Femenino & $\begin{array}{l}\text { Asiste a la escuela dos años y Escuela } \\
\text { Secundaria de Adultos }\end{array}$ & \\
\hline Nicolás & 28 & Masculino & Enseñanza obligatoria (ESO) & \\
\hline Paco & 42 & Masculino & & \\
\hline Lucía & 55 & Femenino & Asistió dos o tres años a la escuela & Sí \\
\hline Pepe & 49 & Masculino & Enseñanza obligatoria (EGB) & \\
\hline Encarni & 62 & Femenino & Asistió 6 años a la escuela & \\
\hline Fernando & 22 & Masculino & Enseñanza obligatoria (ESO) & Sí \\
\hline Chary & 39 & Femenino & $\begin{array}{l}\text { Enseñanza obligatoria (ESO) y Escuela de } \\
\text { adultos }\end{array}$ & \\
\hline Daniel & 22 & Masculino & Enseñanza obligatoria (ESO) & Sí \\
\hline Diego & 63 & Masculino & & \\
\hline Felipe & 40 & Masculino & Enseñanza obligatoria (ESO) & \\
\hline Fran & 40 & Masculino & Enseñanza obligatoria (EGB) & \\
\hline Gonzalo & 26 & Masculino & Enseñanza obligatoria (ESO) & \\
\hline Jaime & 32 & Masculino & Enseñanza obligatoria (ESO) & \\
\hline José & 48 & Masculino & Enseñanza obligatoria (EGB) & Sí \\
\hline Julia & 32 & Femenino & Enseñanza obligatoria (ESO) & \\
\hline Manuel & 43 & Masculino & $\begin{array}{l}\text { Enseñanza obligatoria (EGB) y Forma- } \\
\text { ción Profesional }\end{array}$ & Sí \\
\hline
\end{tabular}




\begin{tabular}{|l|c|l|l|c|}
\hline Marcos & 43 & Masculino & Enseñanza obligatoria (EGB) & Sí \\
\hline Mario & 50 & Masculino & Enseñanza obligatoria (EGB) & \\
\hline Miriam & 41 & Femenino & Enseñanza obligatoria (EGB) & Sí \\
\hline Pablo & 35 & Masculino & Enseñanza obligatoria (EGB) & \\
\hline Saray & 43 & Femenino & Enseñanza obligatoria (EGB) & Sí \\
\hline Valeria & 28 & Femenino & Enseñanza obligatoria (ESO) & \\
\hline
\end{tabular}

${ }^{1} \mathrm{La}$ ausencia de datos en la tabla significa que los participantes no han sabido o querido contestar o no recuerdan la respuesta.

\subsection{Recogida de datos}

En este estudio consideramos a los participantes como coinvestigadores. Estos tuvieron un papel activo en el proceso de recogida de la información y en el análisis de los datos. El instrumento de recogida de datos fue una entrevista.

En primer lugar, para elaborar este instrumento comenzamos con una reunión inicial con los participantes donde explicamos qué era una investigación y cómo se realizaba. Además, expusimos los objetivos y el alcance del presente estudio. En esta reunión hablamos sobre la experiencia de los participantes en las escuelas. Esto sirvió para iniciar a los participantes en el tema de la investigación. Después hicimos una lluvia de ideas en una pizarra sobre los temas que consideraban importante estudiar y, a partir de ahí, las posibles preguntas de las entrevistas. De esta lluvia de ideas derivaron temas: perfil del profesorado, acoso escolar, ajustes curriculares, aulas de apoyo y otras personas importantes en sus procesos de aprendizaje en la escuela. A partir de esta lluvia de ideas, los coinvestigadores y las investigadoras realizaron las preguntas del guion de la entrevista semiestructurada. Todas las preguntas fueron escritas en una pizarra. La única tarea realizada por la investigadora fue la unificación de las preguntas de ambos centros. Esto se hizo eliminando todas las preguntas duplicadas y ordenando las restantes para asegurar la coherencia. Finalmente, el guion estuvo compuesto de 30 preguntas. La entrevista no fue validada por expertos y la investigadora y los investigadores principales fueron los únicos involucrados. Algunas preguntas de la entrevista fueron: $¿ E l$ profesorado se adaptaba a ti en los contenidos que daba? ¿Cómo lo hacía? ¿Piensas que era útil? ¿Te ha servido lo que aprendiste en la escuela? ¿Cómo eran los exámenes? ¿Te ayudaban tus compañeros? ¿Crees que tenías buenos amigos/as en la escuela? ¿Has vivido alguna situación desagradable con tus compañeros? ¿Podrías describirla? ¿Crees que sufriste discriminación? ¿Qué hacías en el recreo?

Realizamos entrevistas individuales. La recogida de la información fue en lugares cercanos para los participantes (todas las entrevistas fueron en sus centros ocupacionales) (Walmsley y Johnson, 2003). Las transcripciones fueron devueltas a los participantes para ser revisadas y poder modificar, eliminar o ampliar la información. 
Algunos participantes revisaron sus entrevistas de forma autónoma y otros fueron ayudados por profesionales del centro.

\subsection{Análisis de datos}

El análisis de los datos fue realizado por un equipo de trabajo formado por la primera autora de este artículo y los participantes de este estudio. En ambos centros ocupacionales se llevó a cabo un taller de dos sesiones (una hora cada sesión). En la primera sesión, explicamos los conceptos básicos de la investigación (definición, objetivos, fases y agentes) y resolvimos dudas de los participantes. La segunda se centró en el análisis de las entrevistas. Estas sesiones requerían un trabajo previo de la investigadora, ya que las entrevistas fueron filtradas y convertidas en tarjetas para facilitar el análisis de datos. El proceso seguido fue:

- Eliminación de los datos personales (p. ej., la oración "Estudié en el colegio San Antonio en Sevilla” es sustituida por "Estudié en el colegio de __ en __”).

- Transformación de oraciones de complejidad morfosintáctica en oraciones simples. (p. ej., la oración "Yo en el colegio aprendí, pero no mucho. Yo he aprendido más en la asociación en la que estaba antes, y aquí, en la asociación en la que estoy ahora" es sustituida por "Yo he aprendido más en los centros ocupacionales que en el colegio").

- Edición de palabras difíciles a palabras fáciles (p. ej., la oración "Yo tenía adaptaciones curriculares y, entonces, yo entendía mejor el temario" es sustituida por "Los profesores adaptaban el tema a mi nivel. Así yo podía entenderlo todo").

- Eliminación de ideas repetidas (p. ej., la frase "Mi madre fue a hablar con el maestro. Ella tuvo que hablar con él para que no me pegaran más. Ella le explicó a mi maestro que me pegaban los compañeros en la escuela. Ella fue la que se lo dijo" es sustituida por "Mi madre informó al maestro de que me pegaban mis compañeros en la escuela”.

- Eliminación de palabras que tienen función fática en el lenguaje y carecen de significado (p. ej., "Vale, te entiendo").

- Sustitución de siglas por palabras (p. ej., la oración “I: ¿Qué edad tienes?” es sustituida por "Investigadora: ¿Qué edad tienes?”).

La segunda sesión consistió en el análisis grupal de cada entrevista. Asignamos al azar tarjetas anónimas a cada participante. Cada participante debía leer la tarjeta asignada al resto de sus compañeros y debatir si su tarjeta era considerada una barrera o una ayuda. Una vez que llegábamos a un acuerdo, colocábamos la tarjeta en un mural en la pared. En el mural identificamos dos categorías: "barreras" y “ayuda”. Estas dos categorías, que coinciden con la fundamentación teórica, fueron consensuadas con los participantes en el estudio. Para facilitar la comprensión de ambos conceptos dibujamos en el mural un símbolo de un tick de color verde junto a la palabra "ayuda" y el símbolo de una equis en rojo para la palabra "barreras". El debate colectivo originó 
nuevas barreras y facilitadores que fueron escritos en el mural con un rotulador. El sistema de categoría y códigos puede consultarse en la Tabla 2.

\begin{tabular}{|c|c|c|}
\hline \multicolumn{3}{|c|}{ TABLA 2. Resultados } \\
\hline & Ayudas & Barreras \\
\hline El profesorado & $\begin{array}{l}\text { - Habilidades personales y pro- } \\
\text { fesionales: empáticos, amables, } \\
\text { comprensivos, motivadores }\end{array}$ & $\begin{array}{l}\text { - Ausencia de compromiso con el } \\
\text { aprendizaje del alumnado } \\
\text { - Disciplinarios e impacientes en el } \\
\text { proceso de enseñanza y aprendizaje }\end{array}$ \\
\hline $\begin{array}{l}\text { Elementos del } \\
\text { currículo }\end{array}$ & $\begin{array}{l}\text { - Ajustes de accesibilidad cog- } \\
\text { nitiva } \\
\text { - Disposición en lugares fijos en } \\
\text { el aula y cercanos a la pizarra } \\
\text { - Ajuste del examen a las carac- } \\
\text { terísticas del alumnado } \\
\text { - Reconocimiento del esfuerzo }\end{array}$ & $\begin{array}{l}\text { - Metodologías magistrales y memo- } \\
\text { rísticas } \\
\text { - Contenido y actividades generali- } \\
\text { zados y homogéneos para todo el } \\
\text { alumnado } \\
\text { - Ausencia de contenidos funcionales } \\
\text { y prácticos (habilidades básicas para } \\
\text { la vida o habilidades sociales) } \\
\text { - El aula de apoyo (contribuye a la ex- } \\
\text { clusión y al etiquetaje) }\end{array}$ \\
\hline Los iguales & $\begin{array}{l}\text { - Ayuda en las tareas escolares } \\
\text { - Defensa y protección ante dis- } \\
\text { criminación y acoso de otros } \\
\text { compañeros }\end{array}$ & $\begin{array}{l}\text { - Discriminación y acoso escolar (en el } \\
\text { aula y en el recreo) } \\
\text { - Ausencia de amigos }\end{array}$ \\
\hline $\begin{array}{l}\text { Directores, } \\
\text { psicólogos y } \\
\text { pedagogos }\end{array}$ & $\begin{array}{l}\text { - Ajuste del proceso de enseñan- } \\
\text { za y aprendizaje a las caracte- } \\
\text { rísticas del alumnado con DI } \\
\text { - La lucha contra el acoso escolar }\end{array}$ & \\
\hline Familia & $\begin{array}{l}\text { - Apoyo emocional } \\
\text { - La lucha contra el acoso escolar }\end{array}$ & \\
\hline
\end{tabular}

\section{Resultados}

\section{1. ¿Cómo el profesorado contribuye a la inclusión del alumnado con discapacidad intelectual?}

Cómo eran los docentes que contribuían a la inclusión fue una primera cuestión que se analizó en este estudio. Los participantes definieron tanto cualidades profesionales como personales que describían a aquellos docentes que habían facilitado su aprendizaje y participación. Estos se caracterizaban por su compromiso con la enseñanza y la persistencia por que aprendiera el alumnado. Los estudiantes valoraban a los docentes que trataban en privado temas delicados, empatizaban, motivaban 
y se preocupaban por las personas más vulnerables. Estos profesionales les dejaron huella, ya que incluso, en algunos casos, después de haber transcurrido muchos años, eran capaces de recordar los nombres y apellidos de los docentes que les trataron con cariño y amabilidad.

Cristina: Si tenía cualquier problema la profesora me apoyaba. Me sentía muy cómoda y relajada con ella. Me ayudaba con las adaptaciones y me decía "si no lo entiendes te lo vuelvo a explicar”. Eso me motivaba. Recuerdo incluso cómo se llamaba. Era muy buena profesora.

Sin embargo, también los participantes tenían claro cómo eran los docentes que no favorecieron su aprendizaje. Los definieron como profesionales poco comprometidos con la enseñanza del alumnado con DI y poco dispuestos a dar respuesta a sus necesidades.

Pepe: Los maestros explicaban la lección una vez o dos. Si no me enteraba pues ya no lo volvían a explicar. Así me fui quedando atrás.

Describieron docentes disciplinarios e impacientes que obstaculizaron su proceso de aprendizaje y se preocupaban solo por aquellos estudiantes con un rendimiento académico alto. En ocasiones, algunos participantes comentaron que algunos docentes no les trataban bien $y$, a veces, incluso cuestionaban su valía.

Lola: Solo atendían a los más listos de la clase. Por mí no se preocupaban. Una maestra me dijo que no servía para nada.

\section{2. ¿Qué elementos del currículo facilitan o dificultan el aprendizaje y participación del alumnado con discapacidad intelectual?}

Los participantes en el estudio describieron diferentes aspectos del currículo que contribuían a su inclusión. En primer lugar, mencionaron la metodología. La mayoría de los estudiantes describieron clases magistrales en las que el profesor era la figura principal de las lecciones. Algunos describieron actividades en grupos y lúdicas como algo excepcional, puesto que no las realizaban diariamente. Muchos contenidos debían ser adquiridos mediante el aprendizaje memorístico, lo que dificultaba aún más su proceso de enseñanza y aprendizaje.

Algunos docentes adaptaron sus clases a las necesidades del alumnado. Ajustaron el contenido para una mejor comprensión, cambiaron el formato y la letra de los materiales para mejorar la accesibilidad de textos o promovieron el aprendizaje visual mediante imágenes que complementaban textos.

Sin embargo, otros participantes indicaron que en el aula ordinaria no ajustaron el contenido a sus necesidades. Los contenidos y las actividades eran homogéneos para todo el alumnado. Esto provocaba que el alumnado con DI tuviera menos oportunidades de aprendizaje que el resto de sus compañeros. 
Pepe: Todos hacíamos lo mismo. No me adaptaron nada. Parecía que hablaban en chino porque yo no comprendía nada. Y después, había que estudiárselo de memoria, así que suspendía los exámenes.

Los estudiantes comentaron haber aprendido contenidos como lectoescritura y conceptos básicos en matemáticas. Sin embargo, recordaron muchas horas en clase en las que no comprendían las explicaciones del profesor y quedaban excluidos de estas. Por ello, algunos habían vivido estas clases como una "pérdida de tiempo en la escuela”. La mayoría resaltó que los contenidos funcionales y prácticos los aprendieron en otros cursos de formación posteriores y no en la enseñanza obligatoria.

José: Lo que yo aprendí en el colegio me sirvió. Aprendí a leer, escribir, hacer cuentas. Pero realmente en el curso de Formación Profesional aprendí cosas más útiles porque me ayudó para desenvolverme en la vida. Por ejemplo: cómo rellenar el cheque de un banco.

Otros participantes echaron de menos en la escuela haber aprendido contenidos relacionados con las relaciones interpersonales para aprender a hacer amigos o entrenar las habilidades sociales.

Marcos: Aprendí cosas en la escuela, pero no aprendí a hacer amigos.

En cuanto a la evaluación, los exámenes que estuvieron ajustados a los estudiantes, tanto en contenido como en formato, fueron considerados como un elemento facilitador. Además, se mostraron a favor de que el esfuerzo fuera considerado un criterio más de la evaluación.

Luis: Una asignatura la tenía suspensa, pero valoraron mi esfuerzo y me aprobaron. Eso estaba bien.

No obstante, consideraron que la mayoría de las veces la evaluación se realizaba mediante exámenes que no estaban adaptados en contenido y formato. Esto provocaba desmotivación, ansiedad y su consecuente experiencia negativa con los exámenes.

María: Los exámenes eran iguales para todo. Ahí era donde yo fallaba. No comprendía nada y suspendía.

Con relación a los espacios, destacar cómo la disposición física del aula podía facilitar el aprendizaje. Los estudiantes mostraron preferencia por ocupar siempre los mismos lugares en el aula y por estar sentados en mesas cercanas a la pizarra para mejorar su atención hacia el docente.

Juan: Me gustaba sentarme delante, pero no por tener discapacidad sino porque prestaba más atención ahí. 
En el caso concreto del aula de apoyo, la mayoría la describió como un elemento favorecedor puesto que en esta aula todos los elementos del currículo estaban adaptados al alumnado. Además, los docentes de esta aula tenían las cualidades docentes descritas anteriormente (empatía, paciencia, amabilidad) y ajustaban los contenidos y la evaluación a las características del alumnado. Muchos destacaron la ausencia de exámenes en el aula de apoyo, algo que les hacía sentirse liberados de la presión que provocaba este tipo de evaluación.

Javi: Cuando salía al aula de apoyo entendía lo que explicaba la profesora. Me sentía relajado y no sentía discriminación. El aula de apoyo para mí era como "Gloria Bendita".

Sin embargo, algunos participantes no estuvieron a favor de asistir a un aula distinta ya que lo consideran un motivo de exclusión. Destacaron, por ejemplo, que en esta aula el material adaptado estaba en blanco y negro ya que eran fotocopias de otros libros y estaban privados de los colores que tenían los libros de texto de sus compañeros. Además, el hecho de tener otro material distinto al del aula ordinaria a algunos participantes les provocaba estrés.

Chari: No me gustaba ir. Me sentía inferior a los demás niños. Además, tenía que cambiar de libro y de cuaderno y era un estrés.

\section{3. ¿Cómo los compañeros dificultan o facilitan la inclusión del alumnado con disca- pacidad intelectual?}

Algunos de los participantes mencionaron a sus compañeros como agentes facilitadores de su proceso de enseñanza y aprendizaje en la escuela. Los iguales les ayudaron con las tareas de clase o la explicación de los contenidos que no comprendían. También destacaron que tuvieron compañeros sin discapacidad que los defendieron ante alguna situación de discriminación. Sin embargo, los participantes relacionaban la amistad exclusivamente con el juego o la ausencia de discriminación durante el juego. No se describieron otras características de la amistad como honestidad, confianza o fidelidad.

Investigadora (I): ¿Crees que tenías buenos amigos en la escuela?

Carmen: Sí, porque jugaban conmigo y no me discriminaban.

Aunque los iguales, en ocasiones, actuaban como ayuda, en otras supusieron una barrera para los estudiantes con DI. Esto fue así principalmente por la discriminación y el acoso escolar que sufrieron algunos participantes. Por un lado, algunos describieron insultos y agresiones verbales y físicas de sus compañeros por tener DI.

Miriam: Me pusieron un mote: “el saltamontes”, porque yo no entendía lo que la maestra explicaba y siempre estaba levantando la mano como un saltamontes. 
Tanto era así que algunos tomaron sus propias medidas para evitar este tipo de discriminaciones. Medidas como intentar pasar desapercibido o, incluso, querer repetir curso para cambiar de compañeros.

Manuela: Intentaba sentarme en los asientos de detrás de la clase para no llamar atención y que no se metieran conmigo.

En concreto, el recreo fue recordado como un lugar marcado por la discriminación con sus iguales, que abarcaba desde el hecho de quedar excluidos en los juegos hasta las agresiones verbales y/o físicas.

Carmen: En el recreo me comía el bocadillo, jugaba e intentaba que no me pegaran.

Estas vivencias negativas les influyeron en sus recuerdos sobre sus experiencias escolares, enfatizando el acaso escolar sufrido.

I: ¿Qué recuerdos tienes de la escuela?

Pepe: Tengo más recuerdos malos que buenos. En la escuela, tú sabes, insultan, me decían: "tonto", "retrasado"...

La mayoría de los participantes consideraron que no eran aceptados entre sus iguales. Sin embargo, desconocían el motivo por el cual tenían dificultades para hacer amigos. Muchos reconocieron que actualmente, en el centro ocupacional, sí eran capaces de hacer amigos y conservarlos.

Luis: Yo en la escuela no era popular. No hacía amigos, no sé por qué. Sin embargo, aquí sí tengo muchos amigos.

\section{4. ¿Qué otros agentes contribuyen a la inclusión del alumnado con discapacidad intelectual?}

Por último, en el estudio se identificaron otros agentes que actuaron como facilitadores en sus trayectorias educativas. Por un lado, nombraron a directores, psicólogos y pedagogos que promovían el ajuste del proceso de enseñanza y aprendizaje y la lucha contra el bullying. Por otro lado, la familia de los participantes también fue considerada un elemento de ayuda. En casa, los familiares les ayudaron con los deberes y les apoyaban con la prestación de recursos como clases particulares fuera de la escuela. Además, la familia ofrecía apoyo emocional a los estudiantes e incluso ejercían de mediadores entre la escuela y el alumno. En algunos casos, las familias alertaron a la escuela de situaciones de bullying entre iguales o de discriminaciones por parte de los docentes.

Cristina: Mi madre fue a hablar con los profesores para que me adaptaran el temario. Mi abuela y mi madre y decían: "Tú vales mucho. Sigue adelante". 


\section{Conclusiones y discusión}

La inclusión de estudiantes con DI en las escuelas ordinarias supone un desafío para el sistema educativo. Una meta ambiciosa que requiere escuchar testimonios de las voces silenciadas o vulnerables (Messiou, 2006). El propósito de este estudio ha sido estudiar las barreras y ayudas que las personas con DI experimentaron en su trayectoria escolar. Las conclusiones del estudio están organizadas en torno a las cuatro preguntas de investigación.

\section{1. ¿Cómo el profesorado contribuye a la inclusión del alumnado con discapacidad intelectual?}

Cuando el docente celebra la diversidad, contribuye a prevenir los prejuicios y los estereotipos y condena la discriminación o la exclusión, se convierte en un elemento facilitador. En otras investigaciones se ha llegado a resultados similares (Alesech y Nayar, 2019; Boynton y Mahon, 2018). Sin embargo, en nuestro estudio no es frecuente encontrar este perfil docente y, por el contrario, sobresale aquel que muestra su preferencia por el alumnado con calificaciones altas o cuestiona la valía de las personas con DI no solo en cuanto a lo educativo, también con relación a lo personal. En definitiva, en lugar de tender puentes a la inclusión, los docentes, en ocasiones, levantan muros que dificultan la permanencia y éxito de estos estudiantes. Este hecho demuestra que aún existen docentes en las aulas que no reconocen los principios de la educación inclusiva y que todavía queda mucho por hacer en los centros educativos. Además, nos hace cuestionar la formación inicial y permanente del docente y la necesidad de realizar una profunda revisión de estas (Buchner et al., 2020).

\section{2. ¿Qué elementos del currículo facilitan o dificultan el aprendizaje y la participa- ción del alumnado con discapacidad intelectual?}

Casi todos los participantes estuvieron escolarizados en centros en los que la enseñanza estaba basada en lecciones magistrales y metodologías centradas en el docente. Para ellos, este tipo de metodología no favorece el aprendizaje y la inclusión de personas con DI. Sin embargo, recomiendan actividades relacionadas con la exploración, la manipulación y la experimentación, que realizaron de forma esporádica e inusual, como acciones eficaces para la adquisición de aprendizajes significativos. Por tanto, las metodologías activas en las que el alumnado es protagonista del proceso de enseñanza y se respetan los ritmos de aprendizaje son facilitadoras de la inclusión (Kurt, 2016). Además, se destacaron las dinámicas cooperativas como una manera de contribuir y mejorar las relaciones sociales entre iguales (Nieto y Moriña, 2019).

En este estudio se identifican barreras relacionadas en el contenido. Los participantes demandan contenidos relacionados con habilidades sociales o contenidos funcionales que pueden facilitar su inserción laboral e inclusión social. Es necesaria, por 
tanto, una reflexión sobre los contenidos curriculares que se imparten en las escuelas y que ponen en evidencia la necesidad de otros contenidos que son fundamentales en la educación de las personas y que han aparecido en estudios previos: por ejemplo, educación emocional (Smith y Low, 2013), habilidades sociales (Díaz-Garolera et al., 2019) o habilidades para la vida independiente (Verdugo y Rodríguez, 2012).

Otro hallazgo de este estudio es la sensación reiterada de "pérdida de tiempo" que tuvieron los participantes durante su escolarización. Estudiar contenidos teóricos o poco funcionales para la vida es percibido como una forma de desaprovechar las oportunidades que el sistema educativo puede dar a los estudiantes con DI (Nota et al., 2018; Scharenberg et al., 2019).

En cuanto a la evaluación, la presencia de exámenes es motivo de ansiedad y desmotivación en el alumnado con DI. En este sentido, sería conveniente, como proponen Datta (2013) y Soulis y Floridis (2010), que la evaluación fuera algo más que rendir cuentas a través de una prueba y que se complementaría con otros instrumentos, como la observación docente o las actividades realizadas por los estudiantes

Entre las ayudas, se hace especial mención al maestro de apoyo. Esta figura fue descrita como una potente ayuda para los estudiantes con DI por realizar un adecuado ajuste curricular y poseer habilidades favorecedoras como la confianza, la paciencia y la motivación de logro hacia los estudiantes. Tales habilidades también fueron destacadas en el estudio de Boynton y Mahon (2018). Sin embargo, algunos participantes vieron este recurso como un elemento de exclusión pues actuaba en contra del principio de normalización y en consonancia con el modelo terapéutico (Sandoval et al., 2019). Estos resultados coinciden con el de Riitaoja et al. (2019).

\section{3. ¿Cómo los compañeros dificultan o facilitan la inclusión del alumnado con disca- pacidad intelectual?}

La actitud de los iguales fue identificada tanto como barrera cuanto como ayuda en las trayectorias escolares de los participantes en el estudio. La discriminación, el acoso escolar o la falta de comprensión y ayuda por parte de los compañeros sin DI supusieron una barrera que no contribuyó a que los estudiantes con DI experimentaran un sentimiento de pertenencia al grupo y, por tanto, sentían que no formaban parte del aula (Rose et al., 2016; Young et al., 2016).

Como se puede concluir en este estudio, los iguales son una pieza clave en la inclusión de los estudiantes con DI. Cuando los compañeros sin discapacidad facilitan el aprendizaje y el bienestar de las personas con DI, mostrando su ayuda en las tareas o evitando situaciones de acoso o discriminación, los estudiantes con DI se muestran motivados por aprender y sienten que forman parte de una institución en la que cada persona es impresionable y es valorado (Scharenberg et al., 2019; Wang y Eccles, 2012).

La ausencia de amistad podría verse también como una barrera para estudiantes con DI. La amistad para los participantes de este estudio consistía únicamente en la existencia de juego. Este hallazgo coincide con el estudio de Robinson et al. (2020). 
Sin embargo, esto no es suficiente para Nepi et al. (2015), quienes afirman que la amistad tiene lugar cuando se establecen relaciones más significativas. Por ello, es recomendable que la escuela tenga presente esta realidad y se desarrollen acciones que promuevan las relaciones significativas del alumnado con DI con el resto de estudiantes (Díaz-Garolera et al., 2019).

\section{4. ¿Qué otros agentes contribuyen a la inclusión del alumnado con discapacidad intelectual?}

Otros agentes de los centros, como directores, psicólogos y pedagogos, fueron facilitadores del proceso de enseñanza y aprendizaje, promoviendo el ajuste curricular y luchando contra el acoso escolar. También la familia supuso una ayuda para las personas con DI en su trayectoria escolar, fundamentalmente por el apoyo emocional ante situaciones de exclusión en la escuela. Esto refuerza la importancia de la cooperación de estas con el contexto educativo para promover el desarrollo integral de las personas con DI (Sandoval et al., 2019; Sharma et al., 2017).

En definitiva, las principales barreras y ayudas fueron identificadas en la práctica docente y en la relación con los iguales. Los hallazgos descritos en este estudio implican diferentes reflexiones y una de ellas está relacionada con la figura del docente y su formación tanto inicial como permanente (Florian, 2014). Aunque las nuevas políticas están realizando una reestructuración de los planes de formación inicial, en la escuela habita una ideología persistente que sigue reproduciendo una enseñanza separatista dentro de un sistema escolar meritocrático (Buchner et al., 2020). Con este estudio, basado en el análisis de las barreras y ayudas descritas por las personas con DI durante su escolaridad, se evidencia la responsabilidad de docentes e iguales como piezas clave en inclusión educativa. Se visibiliza, por tanto, la necesidad de que ambos agentes se conviertan en cómplices de la inclusión de personas con DI en las escuelas.

\section{Limitaciones e investigaciones futuras}

El amplio rango de edad de nuestra muestra (18-63 años) fue una de las limitaciones del estudio ya que encontramos variaciones en las trayectorias escolares debido a las diferentes políticas presentes en el sistema educativo durante la escolaridad de los participantes. Este hecho también podría dar lugar a recuerdos distorsionados de la realidad por el tiempo transcurrido desde que estuvieron escolarizados en los centros educativos.

Otra limitación está relacionada con la edición de tarjetas para realizar el análisis de la información ya que, con estas ediciones, podríamos haber afectado la transparencia e interpretación de los resultados. Sin embargo, esto era necesario, ya que permitía la participación activa en la interpretación de los datos.

La investigación futura podría abordar otros temas de investigación propuestos por personas con DI como el bullying o la formación profesional en la escuela. A 
BARRERAS Y FACILITADORES PARA LA INCLUSIÓN EDUCATIVA

DE PERSONAS CON DISCAPACIDAD INTELECTUAL

C. NIETO CARMONA Y A. MORIÑA DÍEZ

pesar de las limitaciones, creemos que el presente estudio contribuye a la literatura en el ámbito de la educación inclusiva a través de las aportaciones de uno de los grupos más vulnerables y silenciados del sistema educativo: las personas con DI.

\section{Referencias bibliográficas}

Acuña, L., Cabrera, V., Medina, D. y Lizarazo, F. (2017). Necesidades de la familia y de la escuela en la educación de niños con discapacidad intelectual. I+D Revista de Investigaciones, 9(1), 126-137. https://doi.org/10.33304/revinv.v09n1-2017012

Ainscow, M. (2004). Desarrollo de escuelas inclusivas. Ideas, propuestas y experiencias para mejorar las instituciones escolares. Narcea.

Ainscow, M., Slee, R. y Best, M. (2019). Editorial: the Salamanca statement: 25 years on. International Journal of Inclusive Education, 23(7-8), 671-676. https://doi.org/10.1080/1360 3116.2019.1622800

Alesech, J. y Nayar, S. (2019). Teacher strategies for promoting acceptance and belonging in the classroom: a New Zealand study. International Journal of Inclusive Education. https:// doi.org/10.1080/13603116.2019.1600054

ApA. (2017). Ethical principles of psychologists and code the conduct. American Psychological Association. https://www.apa.org/ethics/code/ethics-code-2017.pdf

Boynton, L. y Mahon, J. (2018). Secondary teachers' experiences with students with disabilities: examining the global landscape. International Journal of Inclusive Education, 22(3), 306-322. https://doi.org/10.1080/13603 116.2017.1364793

Buchner, T., Shevlin, M., Donovan, M.-A., Gercke, M., Goll, H., Šı̌̌Ka, J., JanyŠková, K., Smogorzewska, J., Szumski, G., Vlachou, A., Demo, H., Feyerer, E. y Corby, D. (2020). Same progress for all? Inclusive education, the United Nations Convention on the Rights of Persons with Disabilities and students with intellectual disability in European countries. Journal of Policy and Practice in Intellectual Disabilities. https://doi.org/10.1111/ jppi.12368

DAtTA, P. (2013). “Is Test Anxiety a peril for students with intellectual disabilities?”. Journal of Intellectual Disabilities, 17(2), 121-132. https://doi.org/10.1177/1744629513484667

Díaz-Garolera, G., Pallisera, M. y Fullana, J. (2019). Developing social skills to empower friendships: design and assessment of a social skills training programme. International Journal of Inclusive Education. https://doi.org/10.1080/13603116.2019.1625564

Díez, E. y SÁnchez, S. (2015). Diseño Universal para el Aprendizaje como metodología docente para atender a la diversidad en la universidad. Aula Abierta, 43, 87-93. https://doi. org/10.1016/j.aula.2014.12.002

EcheitA, G. (2017). Educación inclusiva. Sonrisas y lágrimas. Aula Abierta, 46, 17-24. https:// doi.org/10.17811/rifie.46.2017.17-24

European Agency For Special Needs And Inclusive Education. (2018). Evidence of the link between inclusive education and social inclusion: a review of the literature. https:// www.european-agency.org/resources/publications/evidence-literature-review

Farmer, T. W., Dawes, M., Hamm, J. V., Lee, D., Mehtaji, M., Hoffman, A. S. y Brooks, D. S. (2018). Classroom social dynamics management: why the invisible hand of the teacher matters for special education. Remedial and Special Education, 39, 177-192. https://doi. org/10.1177/0741932517718359

Ediciones Universidad de Salamanca / CC BY-NC-ND

Siglo Cero, vol. 52 (4), 2021, octubre-diciembre, pp. 29-49 
Florian, L. (2014). What counts as evidence of inclusive education? European Journal of Special Needs Education, 29(3), 286-295. https://doi.org/10.1080/08856 257.2014.933551

Gest, S., Madill, R., Zadzora, K., Miller, A. y Rodkin, P. (2014). Teacher management of elementary classroom social dynamics. Emotional and Behavioral Disorders, 22(2), $107-$ 118. https://doi.org/10.1177/10634 26613512677

GiANGRECO, M. (2017). Expanding opportunities for students with intellectual disability. Educational Leadership, 74(7), 52-57. https://www.ascd.org/publications/educational-leadership/apr17/vol74/num07/Expanding-Opportunities-for-Students-with-Intellectual-Disability.as

Giesbers, S., Hendriks, L., Jahoda, A., Hastings, R. y Embregts, P. (2019). Living with support: experiences of people with mild intellectual disability. Journal of Applied Research in Intellectual Disabilities, 32(2), 446-456. https://doi.wiley.com/10.1111/jar.12542

Gilor, O. y Katz, M. (2018). From normalisation to inclusion: effects on pre-service teachers' willingness to teach in inclusive classes. International Journal of Inclusive Education. https://doi.org/10.1080/13603116.2018.1559365

Jigyel, K., Miller, J., Mavropoulou, S. y Berman, J. (2018). Benefits and concerns: parents' perceptions of inclusive schooling for children with special educational needs (SEN) in Bhutan. International Journal of Inclusive Education. https://doi.org/10.1080/13603116.2018. 1511761

KuntZ, M. y CARTER, W. (2019). Review of interventions supporting secondary students with intellectual disability in general education classes. Research and Practice for Persons with Severe Disabilities, 44(2). https://doi.org/10.1177/1540796919847483

Kurt, X. (2016). Constructivism, dewey, and academic advising. NACADA Journal, 36(2), 1119. https://doi.org/10.12930/ NACADA-14-027

Lacono, T., Keefe, M., Kenny, A. y Mckinstry, C. (2019). A document review of exclusionary practices in the context of Australian school education policy. Journal of Policy and Practice in Intellectual Disabilities, 16(4), 264-272. https://doi.org/10.1111/jppi.12290

LEIVA, J. (2020). La educación intercultural e inclusiva: transformar mentalidades y contextos para el cambio y la innovación educativa. En L. Ortiz y J. J. CARrión (Eds.), Educación inclusiva: abriendo puertas al futuro (pp. 195-202). Dykinson.

López Melero, M. (2011). Barreras que impiden la escuela inclusiva y algunas estrategias para construir una escuela sin exclusiones. Innovación Educativa, 21, 37-54. https://revistas.usc. es/index.php/ie/article/view/23

Low, H. M., Lee, L. W. y Che Ahmad, A. (2018). Pre-service teachers' attitude towards inclusive education for students with Autism Spectrum Disorder in Malaysia. International Journal of Inclusive Education, 22(3), 235-251. https://doi.org/10.1080/13603116.2017.13 62479

Mañas-Olmo, M., González-Alba, B. y Cortés-González, P. (2020). Historias de vida de personas con discapacidad intelectual. Entre el acoso y exclusión en la escuela como moduladores de la identidad. Revista Educación, Politica y Sociedad, 5(1), 60-84. https://hdl. handle.net/10486/689680

Messiou, K. (2006). Understanding marginalisation in education: the voice of children. European Journal of Psychology of Education (Special Issue), 21(3), 305-318. https://doi. org/10.1007/BF03173418

MoriÑA, A. (2010). School memories by young people with disabilities: an analysis of barriers and aids to inclusion. Disability and Society, 25(2), 163-175. https://dx.doi. org/10.1080/09687590903534346 
Nepi, L. D., Fioravanti, J., Nannini, P. y Peru, A. (2015). Social acceptance and the choosing of favourite classmates: a comparison between students with special educational needs and typically developing students in a context of full inclusion. British Journal of Special Education, 42(3) 319-337. https://doi.wiley.com/10.1111/1467-8578.12096

Nieto, C. y Moriña, A. (2019). The dream school: mind-changing perspectives of people with intellectual disabilities. Journal of Applied Research in Intellectual Disability, 32, 15491557. https://doi:10.1111/JAR.12650

NIND, M. (2014). What is inclusive research? Bloomsbury.

Nota, L., Ginevra, M. C. y Soresi, S. (2018). School inclusion of children with intellectual disability: an intervention program. Journal of Intellectual and Developmental Disability, 44(4), 439-446. https://doi.org/10.3109/13668250.2018.1428785

ONU. (2019). Empowering children with disabilities for the enjoyment of their human rights, including through inclusive education. https://undocs.org/A/Hrc/40/27

RAMBERG, J. y WATKINs, A. (2020). Exploring inclusive education across Europe: some insights from the European Agency Statistics on inclusive education. FIRE: Forum for International Research in Education, 6(1), 85-101. https://doi.org/10.32865/fire202061172

Rittaoja, A., Helakorpi, J. y Holm, G. (2019). Students negotiating the borders between general and special education classes: an ethnographic and participatory research study. European Journal of Special Needs Education, 34(5), 586-600. https://doi.org/10.1080/08856 257.2019.1572093

Robinson, S., Hill, M., Fisher, K. y Graham, A. (2020). Belonging and exclusion in the lives of young people with intellectual disability in small town communities. Journal of Intellectual Disabilities, 24(1), 50-68. https://doi.org/10.1177/1744629518765830

Rose, C., Simpson, C. y ElLis, S. (2016). The relationship between school belonging, sibling aggression and bullying involvement: implications for students with and without disabilities. Educational Psychology, 36(8), 1462-1486. https://doi.org/10.1080/01443 410.2015.1066757

Rushbrooke, E., Murray, C. y Townsend, S. (2014). The experiences of intimate relationships by people with intellectual disabilities: a qualitative study. Journal of Applied Research in Intellectual Disabilities, 27, 531-541. https://doi.org/10.1111/jar.12091

Sanahuja, A., Moliner, O. y Moliner, L. (2020). Organización del aula inclusiva: ¿Cómo diferenciar las estructuras para lograr prácticas educativas más efectivas? Revista Complutense de Educación, 31(4), 407-417. https://doi.org/10.5209/rced.65774

Sandoval, M., Simón, C. y Echeita, G. (2019). A critical review of education support practices in Spain. European Journal of Special Needs Education, 34(4), 441-454. https://doi.org/10. 1080/08856257.2018.1533094

Scharenberg, K., Rollett, W. y Bos, W. (2019). Do differences in classroom composition provide unequal opportunities for academic learning and social participation of SEN students in inclusive classes in primary school? School Effectiveness and School Improvement, 30(3), 309-327. https://doi.org/10.1080/09243453.2019.1590423

Sharma, U., Loreman, T. y Simi, J. (2017). Stakeholder perspectives on barriers and facilitators of inclusive education in the Solomon Islands. Journal of Research in Special Educational Needs, 17(2), 143-151. https://doi.org/10.1111/1471-3802.12375

Shogren, K., Gross, J., Forber-Pratt, A., Francis, G., Satter, A., Blue-Banning, M. y Hill, C. (2015). The perspectives of students with and without disabilities on inclusive schools. Research and Practice for Persons with Severe Disabilities, 40(4), 243-260. https:// doi.org/10.1177/15407 96915583493

Ediciones Universidad de Salamanca / CC BY-NC-ND

Siglo Cero, vol. 52 (4), 2021, octubre-diciembre, pp. 29-49 
Simón, C. y BArrios, A. (2019). Las familias en el corazón de la educación inclusiva. Aula Abierta, 48(1), 51-58. https://doi.org/10.17811/rifie.48.1.2019.51-58

Simón, C., Giné, C. y Echeita, G. (2016). Escuela, familia y comunidad: construyendo alianzas para promover la inclusión. Revista Latinoamericana de Educación Inclusiva, 10(1), 25-42. https://doi.org/10.4067/ S0718-73782016000100003

Smith, S. y Low, S. (2013). The role of social-emotional learning in bullying prevention efforts. Theory into Practice, 52(4), 280-287. https://doi.org/10.1080/00405 841.2013.829731

Smogorzewska, J., Szumski, G. y Grygiel, P. (2019). Theory of mind development in school environment: a case of children with mild intellectual disability learning in inclusive and special education classrooms. Journal of Applied Research in Intellectual Disability, 32, 1241-1254. https://doi.org/10.1111/jar.12616

Soulis, S. y Floridis, T. (2010). Sources of stress for Greek students with intellectual disabilities attending mainstream schools. Support for Learning, 25, 74-80. https://doi.org/10.1111/ j.1467-9604.2010.01443.x

UNESCO. (1994). The Salamanca statement and framework for action on special educational needs. Unesco.

UnEsCO. (2020). Global education monitoring report-inclusion and education: All means all. Unesco.

Valentim, A. y Valentim, J. P. (2019). What I think of school: perceptions of school by people with intellectual disabilities. Disability E Society. https://doi.org/10.1080/09687599.2019. 1702507

Van Den Berg, Y. H. y Stoltz, S. (2018). Enhancing social inclusion of children with externalizing problems through classroom seating arrangements: a randomized controlled trial. Journal of Emotional and Behavioral Disorders, 26, 31-41. https://doi. org/10.1177/1063426617740561

Vega, V., Álvarez-Aguado, I., Spencer, H. y González, F. (2020). Avanzando en autodeterminación: estudio sobre las autopercepciones de personas adultas con discapacidad intelectual desde una perspectiva de investigación inclusiva. Siglo Cero, 51(1), 31-52. https://doi. org/10.14201/scero20205113152

Verdugo, M. Á. y Rodríguez, A. (2012). La inclusión educativa en España desde la perspectiva de alumnos con discapacidad intelectual, de familias y de profesionales. Revista de Educación, 358, 450-470. https://doi.org/10.4438/1988-592X-RE-2011-358-086

Walmsley, J. y Johnson, K. (2003). Inclusive research with people with learning disabilities: past, present and futures. Jessica Kingsley.

WANG, M. T. y ECCLES, J. S. (2012). Social support matters: longitudinal effects of social support on three dimensions of school engagement from middle to high school. Child Development, 83, 877-895. https://doi.org/10.1111/j.1467-8624.2012.01745.x

Wilson, N., Jagues, H., Johnson, A. y Brotherton, M. (2017). From social exclusion to supported inclusion: adults with intellectual disability discuss their lived experiences of a structured social groups. Journal of Applied Research in Intellectual Disabilities, 30, 847858. https://doi.org/10.1111/jar.12275

Young, R., Dagnan, D. y Jahoda, A. (2016). Leaving school: a comparison of the worries held by adolescents with and without intellectual disabilities. Journal of Intellectual Disability Research, 60(1), 9-21. https://doi.org/10.1111/jir.12223 


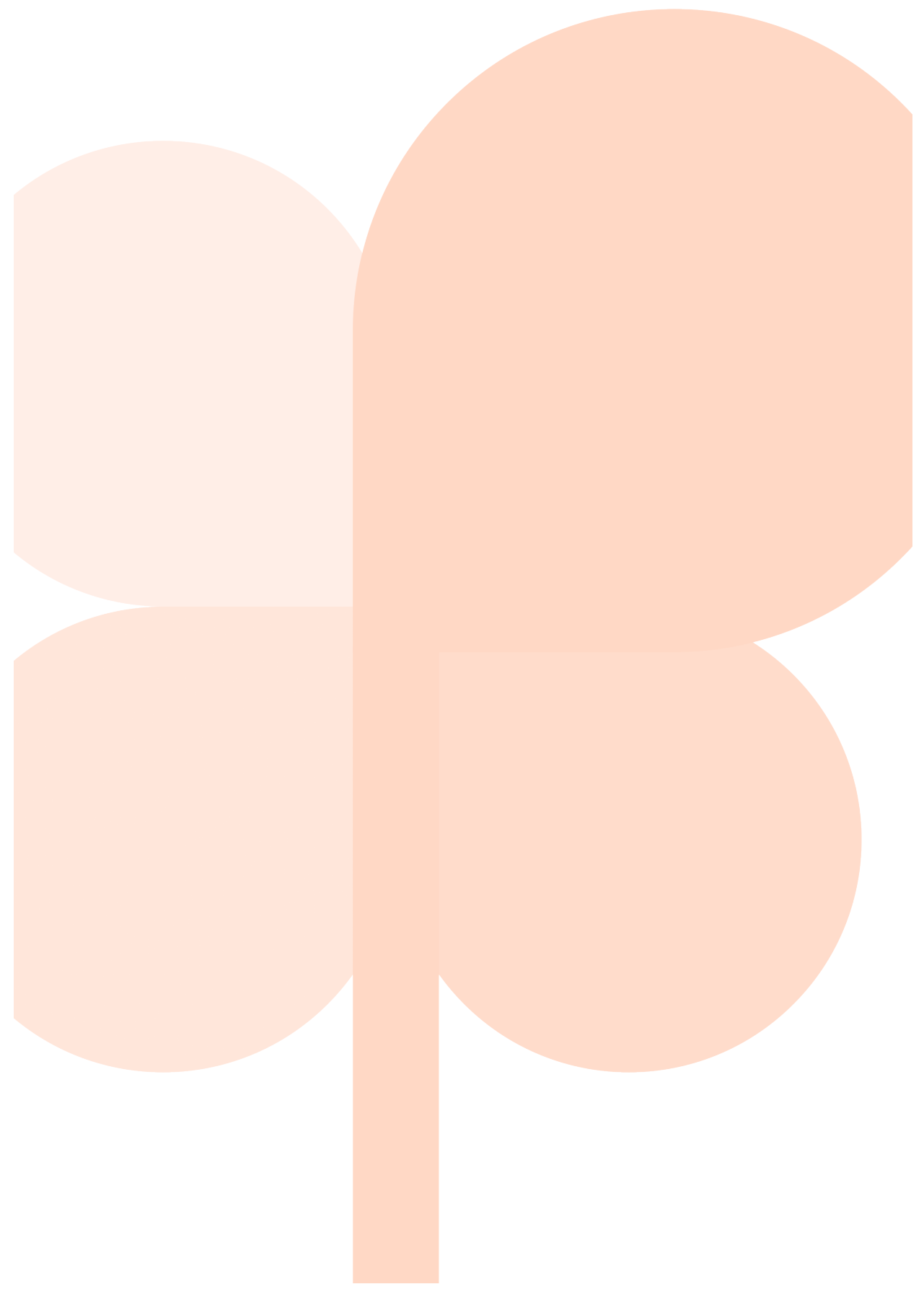

